\title{
Desrespeitos e abusos, maus tratos e violência obstétrica: um desafio para a epidemiologia e a saúde pública no Brasil
}

\author{
Disrespect and abuse, mistreatment and obstetric violence: \\ a challenge for epidemiology and public health in Brazil
}

Tatiana Henriques Leite (https://orcid.org/0000-0002-2861-4480) ${ }^{1}$

Emanuele Souza Marques (https://orcid.org/0000-0002-8633-7290) ${ }^{1}$

Ana Paula Esteves-Pereira (https://orcid.org/0000-0002-0236-2043) ${ }^{2}$

Marina Fisher Nucci (https://orcid.org/0000-0003-3465-9201) ${ }^{3}$

Yammê Portella (https://orcid.org/0000-0002-1955-7779) ${ }^{2}$

Maria do Carmo Leal (https://orcid.org/0000-0002-3047-515X) ${ }^{2}$

${ }^{1}$ Instituto de Medicina Social Hesio Cordeiro, Universidade do Estado do Rio de Janeiro. R. São Francisco Xavier 524, Bloco E, 70 andar, Maracanã 20550-013 Rio de Janeiro RJ Brasil.henriques.tatiana@ gmail.com

${ }^{2}$ Escola Nacional de Saúde Pública Sergio Arouca, Fundação Oswaldo Cruz. Rio de Janeiro RJ Brasil.

${ }^{3}$ Universidade do Estado do Rio de Janeiro. Rio de Janeiro RJ Brasil.

\begin{abstract}
Studies on disrespect and abuse/mistreatment/obstetric violence during pregnancy, childbirth and puerperium have increased in recent decades. However, researchers interested in the subject face many theoretical and methodological difficulties. In this sense, this study aims to discuss and reflect on how issues related to definition and terminology, measurement, and public policies in Brazil have hindered research on this topic and the mitigation of these acts. The first problem addressed was the lack of consensus regarding the terminology and definition of this construct. This situation causes a cascading effect, impacting the use of non-validated measurement instruments and, consequently, a lack of accuracy and comparability between studies. Another issue mentioned is the lack of studies exploring the consequences of these acts on women's and newborn's health, which is one of the main gaps on the subject today. The absence of causal studies affects health decision-making, impairing the elaboration of specific public policies.
\end{abstract}

Key words Violence, Violence against women, Pregnancy, Childbirth, Puerperium, Epidemiology
Resumo Estudos sobre desrespeitos e abusos/maus tratos/violência obstétrica durante gestação, parto e puerpério têm aumentado nas últimas décadas. Entretanto, os pesquisadores interessados na temática se deparam com muitas dificuldades teóricas e metodológicas. Nesse sentido, o objetivo do presente estudo consiste em discutir e refletir sobre como questões relacionadas a definição e terminologia, mensuração e políticas públicas no Brasil têm dificultado a pesquisa da temática, assim como a mitigação desses atos. O primeiro problema abordado foi a falta de consenso em relação a terminologia e definição desse construto. Essa situação provoca um efeito em cascata, com a utilização de instrumentos de aferição não validados que implicam falta de precisão e comparabilidade entre os estudos. Outra questão mencionada é a falta de estudos explorando as consequências desses atos na saúde da mulher e do recém-nascido, configurando uma das principais lacunas sobre o tema atualmente. A ausência de estudos causais impacta a tomada de decisão em saúde, prejudicando a elaboração de políticas públicas específicas.

Palavras-chave Violência, Violência contra a mulher, Gestação, Parto, Puerpério, Epidemiologia 


\section{Introdução}

Os Objetivos de Desenvolvimento Sustentável (ODS) reconhecem que o enfrentamento à desigualdade de gênero é de suma importância no combate às diferenças de gênero quanto à oportunidades de estudo, trabalho, renda, participação política, entre outras ${ }^{1}$. Nesse contexto, a confrontação de todas as formas de violência contra a mulher se coloca como uma das temáticas a serem trabalhadas para atingir essa equidade ${ }^{1}$.

A violência contra a mulher está inserida em dois tipos distintos de manifestação de violência: a coletiva e a interpessoal. A primeira contempla os atos perpetrados pelo Estado ou instituições, como violência e escravidão sexual durante guerras e conflitos, violência policial, terrorismo, entre outros. A segunda se refere à violência praticada por indivíduos com ou sem vínculo pessoal/íntimo com a vítima. Pode ocorrer dentro do domicílio ou na comunidade, e inclui violência doméstica (psicológica, física e sexual), coerção reprodutiva, assédio sexual, estupro, mutilação genital, entre outras ${ }^{2}$.

De acordo com alguns pesquisadores do tema, sociedades cujas culturas aceitam e toleram a violência contra a mulher estão mais propensas a naturalizar esses atos, inclusive aqueles que ocorrem dentro dos serviços de saúde ${ }^{3}$. Não obstante, publicações recentes têm demonstrado que muitas mulheres no mundo sofrem maus tratos, desrespeitos, abusos ou mesmo violência durante a gestação, o parto e o puerpério por parte dos profissionais de saúde $e^{4-6}$. O mesmo é verdade para as mulheres em situação de aborto ${ }^{7}$. No Brasil, segundo estudos hospitalares realizados em 2011 e 2015, a prevalência nesses casos foi de $44,3 \%$, e $18,3 \%$, respectivamente ${ }^{6,8}$. Devido a essa alta magnitude, desrespeitos e abuso, maus tratos e violência durante o parto são considerados uma grave forma de violência de gênero e comprometem os direitos humanos fundamentais das mulheres, além de ser um problema de saúde pública mundial ${ }^{9}$.

Na última década, o interesse por estudar essa forma de violência durante o parto tem crescido, principalmente devido ao movimento feminista e aos grupos que buscam reivindicar os direitos individuais das mulheres, reforçando a sua liberdade e autonomia nesse momento tão especial da vida ${ }^{10,11}$. O aumento da escolaridade das mulheres, a entrada no mercado de trabalho e a conquista de direitos sexuais e reprodutivos tornaram o cenário bastante favorável para ampliar a discussão.
Apesar do aumento do interesse científico e da sociedade civil pela temática, muitas dificuldades teóricas e metodológicas são impostas aos pesquisadores que desejam explorar esse tema, tais como a falta de consenso entre as terminologias e as definições utilizadas, e consequentemente a dificuldade de mensuração do problema, o que refreia a identificação e o conhecimento da magnitude, bem como a realização de estudos causais e sobre as consequências desses atos na saúde materno-infantil, estes últimos escassos na literatura $^{12}$. Sendo assim, o objetivo do presente estudo é, com base na literatura científica existente, discutir questões conceituais e metodológicas que dificultam e restringem a pesquisa dessa temática.

\section{Terminologias e definições}

Não há consenso na literatura acerca da terminologia e da definição que melhor expressem os atos de desrespeitos, abusos, maus tratos e violência contra a mulher perpetrados por profissionais de saúde durante o ciclo gravídico e puerperal. Os termos mais utilizados são disrespect and abuse in facility-based chilbirth ${ }^{13}$; mistreatment of women in childbirth at health facilities ${ }^{14}$; and $o b$ stetric violence ${ }^{10}$. Esses termos são usados muitas vezes como sinônimos. Entretanto, os mesmos apresentam definições, críticas e lacunas distin$\operatorname{tas}^{12}$.

O termo disrespect and abuse in facility-based childbirth foi o primeiro a ser utilizado para endossar situações de desrespeitos, maus tratos e violência perpetrados pela equipe de saúde no ciclo gravídico puerperal. O termo foi proposto por Bowser e Hill ${ }^{13}$ (2010) e contempla sete dimensões em sua definição, a saber: 1) physical abuse; 2) non-consented care; 3) non-confidencial care; 4) non-dignified care (including verbal abuse); 5) discrimination based on specific patient attributes, 6) abandonment of care; 7) detention in facilities. Esse termo e sua definição foram os primeiros a dar visibilidade ao tema em nível mundial, pois serviram de base para uma importante publicação da Organização Mundial de Saúde (OMS), em 2015, intitulada The prevention and elimination of disrespect and abuse during facility-based childbirth ${ }^{9}$. A partir da criação desse termo e sua definição, pesquisas epidemiológicas na área foram estimuladas, com o intuito de identificar e compreender melhor o assunto ${ }^{4,15,16}$, até então pouco estudado.

No entanto, tanto o termo como a sua definição possuem algumas limitações. Destacam-se a 
insuficiência de suas categorias para caracterizar todas as formas de desrespeitos e abusos existentes e a não menção da necessidade de intencionalidade do agente perpetrador ${ }^{17}$. Além disso, essa definição enfatiza que os atos de desrespeitos e abusos são exclusivos a situações de parto e nascimento, desconsiderando que esses eventos também podem ocorrer durante a gravidez (no pré-natal), no pós-parto e em situações de abortamento. Por fim, autores como Savage e Castro ${ }^{12}$ e Bohren e colaboradores ${ }^{18}$ pontuam a dificuldade de operacionalização das dimensões propos$\operatorname{tas}^{12,18}$ o que, para eles, foi o motivo para que não houvesse uma proposta de instrumento para uso em estudos de cunho epidemiológico.

Com o objetivo de solucionar algumas das limitações mencionadas, Bohren et al. ${ }^{14}$ (2015) propuseram um novo termo, mistreatment of women in childbirth at health facilities. Esse termo também apresenta sete dimensões, denominadas de termos de terceira ordem: 1) physical abuse; 2) sexual abuse; 3) verbal abuse; 4) stigma and discrimination; 5) failure to meet professional standards of care; 6) poor rapport between women and providers; 7) health system condition and costraints. Cada termo de terceira ordem é dividido em termos de segunda e primeira ordem. Por exemplo: physical abuse (third order theme) $\rightarrow$ second-order themes: (1) use of force; (2) physical restraint $\rightarrow$ first-order themes: (1) women beaten, slapped, kicked, or pinched during delivery; and (2) women physically restrained to the bed or gagged during delivery ${ }^{14}$.

A definição proposta por Bohren e colaboradores $^{14}$ avança porque enumera uma maior quantidade de atos que são considerados maus tratos, tornando o conceito mais inclusivo do que o anterior. Essa definição permitiu a ampliação de algumas discussões envolvendo o contexto de atenção ao parto e nascimento e a intencionalidade dos atos de maus tratos. Em relação ao contexto, sabe-se que a condição de trabalho dos profissionais de saúde está relacionada com a qualidade do atendimento ofertado às mulheres. Maternidades que operam frequentemente acima de suas capacidades podem deixar muitas mulheres com o sentimento de estarem sendo negligenciadas e tendo a comunicação dificultada com a equipe de saúde. Assim, a condição do sistema de saúde é um elemento importante e que deve ser levado em consideração para explicar possíveis motivações de maus tratos e experiências negativas vivenciadas durante o parto ${ }^{14}$. Somada a essa questão, a falta de estrutura disponível no atendimento à mulher também é um componente importante, uma vez que independe da equipe de saúde, sendo considerada uma violência institucional. Uma estrutura inadequada tem potencial para ferir a dignidade e a privacidade da mulher, assim como reduz a capacidade do hospital/maternidade em ofertar o melhor atendimento possível, considerando as evidências científicas ${ }^{14}$.

Outro ponto mencionado por Bohren e colaboradores diz respeito à intencionalidade dos atos de maus tratos. Os autores reconhecem que esses atos podem ser intencionais ou não, porém não julgam necessário fazer essa distinção, alegando não haver diferença na avaliação do impacto para a mulher e o recém-nascido. No entanto, esse posicionamento pode ser questionado, uma vez que não está de acordo com a definição de violência interpessoal proposta pela OMS². Nessa definição, a violência é descrita como "atos de natureza intencional com potencial de causar danos", associando a intencionalidade ao próprio ato de violência, independentemente do resultado que o mesmo produz ${ }^{2}$. É importante pontuar que tal definição de violência proposta pela OMS leva em consideração os possíveis efeitos negativos da violência na saúde e no bem-estar dos indivíduos. Na falta de consenso sobre termos e definições nessa área, é preciso fazer uma análise crítica a respeito de pontos positivos e negativos de se ter uma definição que leve em consideração atos intencionais e não intencionais. Como ponto positivo, destaca-se a possibilidade de captar experiências negativas sutis e subjetivas. O ponto negativo da incorporação dessa questão consiste na ampliação do escopo de atos incluídos na definição, o que pode dificultar a operacionalização por meio de um instrumento.

Além de ter possibilitado importantes discussões sobre o tema, como as já mencionadas, o principal progresso da proposta de Bohren e colaboradores $^{14}$ foi a criação de um questionário com o objetivo de mensurar mistreatment in childbirth de uma forma padronizada, cujo objetivo principal consiste em estimar a prevalência, permitir uma maior comparação entre os estudos e estimular o desenvolvimento de estudos causais ${ }^{18}$. Desde a criação desse termo e sua definição, a OMS adotou o termo mistreatment em todas as suas publicações ${ }^{3}$.

Outro termo bastante popular, principalmente na América Latina, mas não restrito a esses países, é violência obstétrica. A Venezuela foi pioneira na construção do termo e sua definição na forma de uma legislação, em 2007. A definição venezuelana considera violência obstétrica toda 
conduta, ação ou omissão realizada pela equipe de saúde, de maneira direta ou indireta, em âmbito público ou privado, caracterizada pela "apropriação do corpo da mulher e dos processos reprodutivos pelo profissional de saúde, que se expressa por um atendimento desumanizado, abuso da medicalização e patologização dos processos naturais, resultando em perda de autonomia e capacidade de decidir livremente sobre o seu corpo e a sua sexualidade, afetando negativamente a sua qualidade de vida" ${ }^{19}$. Na Argentina, em 2009, foi aprovada lei que define tanto a violência obstétrica como a violência contra a liberdade reprodutiva. Segundo essa lei, violência obstétrica é definida por "atos exercidos pelos profissionais de saúde sobre o corpo, envolvendo também os processos reprodutivos das mulheres, que podem ser expressas através do tratamento desumanizado, abuso de medicalização e patologização de processos naturais no parto, nascimento e puerpério da mulher e do seu bebê" ${ }^{19}$.

Em seguida, baseados nas propostas da Venezuela e da Argentina, diversos países latino-americanos aprovaram leis específicas abordando o termo violência obstétrica. Em 2013, o mesmo ocorreu no Panamá e na Bolívia ${ }^{19}$. Entre 2007 e 2018, vários estados mexicanos aprovaram leis que definem e punem a violência obstétrica no país. E por fim, em 2017, o Uruguai também aderiu ao uso deste termo ${ }^{19}$. No Brasil, não há uma lei federal específica que aborde o tema violência obstétrica. No entanto, a Constituição Federal contempla de forma subjetiva a proteção contra a ocorrência desse tipo de violência, pois a mesma deixa claro que o Estado deve garantir o direito à saúde, à integridade física e mental e à não discriminação. Outro marco importante foi a convenção de Belém do Pará, quando foi reconhecido o direito de toda as mulheres de viver livre de violência. Para complementar, também é possível citar o código de ética médico que, em seus princípios fundamentais, estabelece sigilo e confidencialidade ao paciente ${ }^{19}$.

Porém, o termo violência obstétrica tem sido rejeitado entre os profissionais de saúde que atuam na assistência ao parto, sobretudo no Brasil. O principal motivo para sua baixa aceitação é a incorporação da patogenização e da medicalização do parto e nascimento à definição. Assim, embora alguns atos possam ser mais facilmente entendidos como violência, outros, reivindicados por ativistas pela humanização do parto, dizem respeito a procedimentos médicos rotineiros. Dessa forma, a denúncia de atos de violência obstétrica, pela perspectiva do movimento social, é um modo de questionar certa visão de mundo a respeito do que constitui ou não a assistência ao parto ideal. $\mathrm{Na}$ tentativa de amenizar esse conflito, mas sem deixar de reforçar a importância de um parto/nascimento respeitoso, a Rede pela Humanização do parto (ReHuNa), por exemplo, tem optado por fazer um contraponto com termos positivos como "humanização do parto" e "a promoção dos direitos humanos das mulheres", em oposição à utilização do termo violência obstétrica ${ }^{10}$.

Algumas evidências empíricas encontradas no Brasil reforçam a hipótese de que a medicalização desnecessária do parto pode não ser interpretada como uma forma de violência pela equipe de saúde e, inclusive, por algumas mulheres. Por exemplo, todos os indicadores de qualidade da atenção ao parto são piores avaliados por mulheres negras e com baixa escolaridade ${ }^{20}$ realizado com base na pesquisa Nascer no Brasil. Foram incluídas 15.688 mulheres entrevistadas no pós-parto, por telefone, de março de 2011 a fevereiro de 2013. Todas as variáveis componentes da relação profissional/parturiente (tempo de espera, respeito, privacidade, clareza nas explicações, possibilidade de fazer perguntas e participação nas decisões. No entanto, algumas intervenções, como episiotomia e cesariana eletiva, são mais frequentes em mulheres brancas e com alta escolaridade ${ }^{21}$, criando um aparente paradoxo. É importante pontuar que, de acordo com a OMS, toques vaginais excessivos e por múltiplos profissionais, episiotomia de rotina, manobra de kristeller, aminiotomia precoce, uso de ocitocina de rotina, cesariana sem indicação clínica e imposição da posição do parto são práticas não recomendadas na atenção ao parto ${ }^{22}$. No entanto, muitos profissionais de saúde no Brasil não entendem essas práticas como abusivas, mas como atos inerentes à atenção ao parto ${ }^{21}$.

Para quebrar esse paradigma, alguns pesquisadores acreditam que é necessário mudar a forma de ensino e aprendizagem dos profissionais que atuam durante o ciclo gravídico-puerperal, enfatizando a abordagem baseada em evidência científica e no respeito a autonomia e dignidade da mulher ${ }^{23}$.

\section{Mensuração}

A falta de consenso na terminologia e na definição empregadas nos atos de desrespeitos, abusos, maus tratos e violências obstétricas, bem como a ausência de um instrumento validado para mensurar esse construto, tornam os 
estudos epidemiológicos complexos e de difícil comparação.

$\mathrm{O}$ instrumento mais popular existente no presente momento foi proposto por Borhen e colaboradores ${ }^{14} \mathrm{em}$ 2015. Ele foi criado e utilizado em alguns estudos desenvolvidos em países africa$\operatorname{nos}^{18}$. Apesar de avançar no processo de mensuração, apresenta limitações importantes. A principal se refere às dimensões (ou termos de $3^{\mathrm{a}}$ ordem) contempladas, que abarcam claramente apenas três das sete dimensões descritas (violência psicológica/verbal, violência física e estigma e discriminação). $O$ instrumento apresenta ainda questões sobre exames vaginais, métodos não farmacológicos para alívio para dor, mobilidade, alimentação, presença de acompanhante durante o trabalho de parto e parto, mas não descreve em qual dimensão essas perguntas estão inseridas. Há, ainda, um bloco de questões enfocando demandas insensatas, estrutura tarifária e negligência, que mistura situações diversas que parecem não estar conectadas diretamente. Por fim, a violência sexual foi completamente excluída desse instrumento.

Borhen e colaboradores ${ }^{14}$ não deixam claro se consideram esse conjunto de questões propostas como um instrumento de fato ou um questionário. Os autores não realizaram nenhuma análise das propriedades psicométricas do instrumento, tampouco apresentaram uma forma de sistematização da informação e, consequentemente, classificação das mulheres em relação à violência vivenciada. Além das limitações mencionadas, o instrumento é considerado inadequado para avaliar possíveis situações de maus tratos contra as mulheres durante o pré-natal e em situação de abortamento.

A ausência de instrumentos válidos e acurados para a mensuração desse problema acarreta impossibilidade de estimar com precisão a prevalência do evento de interesse, falta de comparabilidade entre os estudos existentes em diferentes contextos e populações, dificuldade de monitoramento das estimativas ao longo do tempo e obstáculo a estudos enfocando fatores de risco e consequências desses eventos na saúde da mulher e do recém-nascido.

Um exemplo da falta de precisão na estimação da prevalência e na comparação dos dados pode ser observado ao analisar os dois estudos, o primeiro de base hospitalar e o segundo de base populacional, publicados recentemente no Brasil. Ambos utilizaram o termo disrespect and abuse in childbirth. No primeiro, um inquérito nacional sobre parto e nascimento realizado em 2011/2012, a prevalência de desrespeitos e abusos ficou em torno de $44,3 \%$, considerando conjuntamente sete indicadores que abarcavam a ocorrência de violência física e psicológica, tratamento desrespeitoso, falta de informação, privacidade e comunicação com a equipe de saúde, impossibilidade de fazer perguntas e perda de autonomia ${ }^{24}$. Na segunda pesquisa, porém, utilizando dados da coorte de Pelotas (2015), estimou-se uma prevalência de desrespeitos e abusos em $18,3 \%$, considerando quatro perguntas sobre violência verbal, violência física, realização de intervenções contra a vontade da mulher e negligência. Nesse caso, acredita-se que a diferença de prevalência se deve principalmente às formas distintas de mensuração ${ }^{6}$.

\section{Escassez de estudos que avaliam as consequências de desrespeitos, abusos, maus tratos e violência obstétrica}

A literatura acerca de desrespeitos, abusos, maus tratos e violência obstétrica abarca um número reduzido de estudos com enfoque nas consequências desses atos na saúde da mulher e, em alguns casos, do recém-nascido. Essa escassez é uma das principais lacunas sobre o assunto na atualidade ${ }^{12,2}$. Essa carência é, na realidade, consequência da falta de consenso em relação à terminologia e à definição desse campo teórico e, principalmente, da inexistência de um instrumento validado para captar esse construto de forma mais acurada.

Muitas hipóteses causais são aventadas pelos pesquisadores da área, porém poucas possuem respaldo científico. Algumas pesquisas sugerem que desrespeitos, abusos, maus tratos e violência obstétrica podem estar associados a desfechos negativos na saúde de suas vítimas, como aumento da probabilidade de desenvolvimento de ansiedade, transtorno de estresse-pós-traumático e depressão pós-parto ${ }^{24-26}$, baixa utilização de serviços de saúde pela mulher e recém-nascido no período pós-parto ${ }^{12}$ e maior dificuldade na prática do aleitamento materno ${ }^{12}$. Considerando que um dos componentes de desrespeito, abuso, maus tratos e violência obstétrica é a negligência, desfechos prováveis citados são o aumento da ocorrência de near miss materno e da mortalidade materna e feta ${ }^{10}$. Também é possível que a vivência de violência obstétrica altere a decisão da via de parto em gestações subsequentes (por exemplo: mulheres que sofreram maus tratos durante um parto vaginal podem optar por uma cesariana eletiva em gestações futuras). No entanto, grande parte dos desfechos mencionados são 
baseados em hipóteses, com pouca ou nenhuma evidência científica empírica.

Além disso, vale ressaltar que a ocorrência de desrespeitos, maus tratos e violência obstétrica durante o ciclo gravídico puerperal também pode impactar negativamente em questões mais subjetivas, como a percepção em relação à qualidade da atenção recebida, à satisfação com o parto, entre outras.

Um novo olhar sobre os termos, definições, formas de operacionalização da violência obstétrica

A elaboração de um termo e, consequentemente, uma definição que endosse a maior parte dos atos de desrespeitos, maus tratos e violência perpetrados por profissionais de saúde contra as mulheres durante o ciclo gravídico e puerperal, e que seja aceito pelos pesquisadores e se torne um consenso na área ainda, é um grande desafio. No entanto, essa questão é de suma importância para melhor compreender os fenômenos ligados a esse problema. É necessário delimitar os atos de forma que captem as experiências negativas e subjetivas da vítima. Ao mesmo tempo, não pode ser tão amplo a ponto de perder o significado e marginalizar toda e qualquer ação realizada pela equipe de saúde no atendimento à mulher. Além disso, é preciso demarcar quais atos são perpetrados pelos profissionais de saúde durante a assistência à mulher de fato e quais são decorrentes da falta de investimento em estrutura, materiais e equipamento do setor saúde ${ }^{2}$. Tendo isso em mente, o uso do termo violência obstétrica para caracterizar os atos de violência psicológica/verbal, física, sexual, e negligência intencional pela equipe de saúde no atendimento à mulher no pré-natal, parto, nascimento e em situações de abortamento pode ser bastante vantajoso, uma vez que abrange um escopo delimitado de manifestações que são bem definidas na literatura ${ }^{2}$.

Nesse sentido, Bohren e colaboradores já deram os primeiros passos na elaboração de um instrumento. É importante ressaltar que o questionário proposto por eles engloba três dessas quatro dimensões já consideradas na definição de violência obstétrica. O desenvolvimento de um instrumento poderia facilitar a realização de estudos descritivos e causais, de modo a possibilitar o conhecimento da magnitude do problema, bem como embasar com evidências científicas a elaboração de políticas públicas com o objetivo de mitigar e conduzir esse problema. Ainda assim, o desafio seria a elaboração de itens para a dimensão de violência sexual não contemplada na versão proposta por Bohren et al. ${ }^{14}$ (2015), além da realização de análise psicométrica para avaliação do instrumento em si.

$\mathrm{O}$ uso do termo violência obstétrica restrito a essas quatro dimensões também estaria de acordo com a tipologia utilizada pela OMS para definir essas mesmas manifestações de violência em outros subtipos, tais como da violência interpessoal - que inclui a violência entre parceiros íntimos, violência contra a criança e o adolescente e violência contra o idoso. É importante ressaltar que Bowser e Hill ${ }^{13}$ (2010) e Bohren e colaboradores $^{14}$ (2015) também identificaram essas dimensões em suas respectivas definições de desrespeitos, abusos e maus tratos.

Outro ponto que merece ser pontuado é a utilização do termo obstétrico, em contraponto a childbirth, mais comumente utilizado. Essa decisão evidenciaria que tal tipo de violência pode ocorrer na gravidez, no parto, no puerpério e no aborto, ou seja, em todos os eventos do ciclo gravídico puerperal. É importante mencionar que o uso desse termo também já foi aventado por outros pesquisadores da América Latina ${ }^{23,27}$.

Vale ressaltar que a opção por utilizar o termo violência obstétrica para caracterizar atos de violência e negligência não se opõe à utilização do termo mistreatment. Ambos, bem como suas respectivas definições, podem e devem coexistir. Para tal, é preciso levar em consideração o fato de mistreatment se referir aos episódios de desrespeito e violação de direitos e da dignidade das mulheres, apresentando um significado mais amplo. Desse modo, contempla tanto atos de violência propriamente ditos quanto atos mais sutis. Assim, a violência obstétrica seria apenas um componente desse construto maior denominado mistreatment.

No entanto, não podemos deixar de mencionar que a ressignificação do termo violência obstétrica, sob a perspectiva de facilitar a mensuração e adequar a proposta conceitual da OMS, pode não ser uma unanimidade. Cabe observar que a exclusão da questão da medicalização e patologização do processo de parto e nascimento pode não agradar a todos, uma vez que o assunto circula em diferentes campos discursivos, como os do direito, da saúde e de movimentos feministas ${ }^{28}$. Embora exista diálogo entre eles, cada um desses campos possui racionalidades distintas e irá produzir suas próprias acepções da violência obstétrica, com perspectivas nem sempre coincidentes. A amplitude de entendimentos também pode ser entendido, em certo sentido, como parte de um 
processo de embates e disputas ${ }^{28}$. Optando por uma definição restrita às violências e negligências intencionais, favorecemos a mensuração, porém esvaziamos um termo que, no contexto brasileiro, é muito caro aos movimentos sociais que têm uma pauta combativa à medicalização excessiva $e$ à patologização da assistência ao parto.

Para finalizar, é importante mencionar que, no Brasil, há algumas políticas públicas de melhoria da qualidade da assistência à mulher no pré-natal e no parto com potencial impacto na redução da violência obstétrica, como: Programa Nacional de Humanização do Parto e Nascimento (2000); Lei do Acompanhante (2005), Rede Cegonha - Rede de Atenção Materno Infantil (2011); e Diretriz Nacional de Atenção à Gestante $(2015 / 2016)^{29}$. Além disso, também merece destaque o trabalho de alguns movimentos sociais, com destaque para o $\mathrm{ReHuNa}$, que tornou o termo "parto humanizado" acessível ao público geral, contribuindo para a disseminação de informação por meio de ONGs, redes sociais e filmes $^{23}$. Porém, esses documentos não falam abertamente sobre violência obstétrica e nenhum de seus "sinônimos".

Em 2019, o Ministério da Saúde brasileiro publicou o Ofício no 017/19 - JUR/SEC, julgando o termo violência obstétrica como inadequado e banindo a sua utilização em documentos legais e em políticas públicas. Após essa decisão polêmica, sob recomendação do Ministério Público Federal, o Ministério da Saúde publicou nota reconhecendo o direito legítimo das mulheres em usar o termo violência obstétrica para retratar as experiências de desrespeitos, abusos, maus tratos e violência vivenciadas, bem como o uso de práticas não baseadas em evidências científicas em situações de atenção à saúde ${ }^{30}$. No entanto, os textos oficiais e as políticas de saúde brasileiras seguem não empregando o termo. Essa omissão do Estado pode ser parcialmente explicada pela falta de estatísticas confiáveis sobre a prevalência desses atos na população brasileira e de evidências científicas acerca das consequências maléficas na saúde da mulher e do recém-nascido que vivenciaram alguns desses atos. Por fim, é importante mencionar a resistência dos profissionais de saúde na utilização do termo violência obstétrica.

\section{Considerações finais}

Há muito o que se avançar com relação a pesquisas científicas envolvendo a temática de desrespeito, abusos, maus tratos e violência contra a mulher perpetrados pelos profissionais de saúde no ciclo gravídico puerperal. A falta de consenso quanto à terminologia e à definição desse construto provoca um efeito em cascata, causando falta de precisão na estimação da magnitude desses atos, dificuldade de comparabilidade entre diferentes estudos e países (distintas definições e, consequentemente, distintas formas de mensuração) e escassez de estudos analíticos enfocando possíveis desfechos negativos desse problema para a saúde e o bem-estar da mulher e do recém-nascido.

A ausência de estudos epidemiológicos causais sobre a temática impacta a tomada de decisão na área da saúde, uma vez que o conhecimento gerado influencia a elaboração de políticas públicas específicas para prevenção desses atos por parte dos gestores de saúde. Diante das questões mencionadas, estudos futuros com foco na criação de um termo e de uma definição consensual na área e, por conseguinte, no desenvolvimento de um instrumento com boas propriedades psicométricas para avaliar situações de desrespeitos e abusos, maus tratos e violência obstétrica durante a gravidez, o parto, o puerpério e em situações de abortamento se fazem necessários.

\section{Colaboradores}

TH Leite e ES Marques: concepção, planejamento, redação do trabalho e aprovação da versão final. AP Esteves-Pereira, MF Nucci, Y Portella e MC Leal: revisão crítica do conteúdo e aprovação da versão final. 


\section{Financiamento}

Coordenação de Aperfeiçoamento de Pessoal de Nível Superior (Capes/PNPD- Fiocruz).

\section{Referências}

1. Nações Unidas no Brasil. Glossário de termos do Objetivo de Desenvolvimento Sustentável 5: alcançar a igualdade de gênero e empoderar todas as mulheres e meninas. Brasília: ONU Brasil; 2016.

2. Krug EG, Mercy JA, Dahlberg LL, Zwi AB. The world report on violence and health. Lancet 2002; 360(9339):1083-1088.

3. Bohren MA, Mehrtash H, Fawole B, Maung TM, Balde MD, Maya E, Thwin SS, Aderoba AK, Vogel JP, Irinyenikan TA, Adeyanju AO, Mon NO, Adu-Bonsaffoh K, Landoulsi S, Guure C, Adanu R, Diallo BA, Gülmezoglu AM, Soumah AM, Sall AO, Tunçalp Ö. How women are treated during facility-based childbirth in four countries: a cross-sectional study with labour observations and community-based surveys. Lancet 2019; 6736(19):1750-1763.

4. Castro R, Frías SM. Obstetric violence in Mexico: results from a 2016 National Household Survey. Violence Against Women 2019; 26(6-7):555-572.

5. Sando D, Kendall T, Lyatuu G, Ratcliffe H, McDonald K, Mwanyika-Sando M, Emil F, Chalamilla G, Langer A. Disrespect and abuse during childbirth in Tanzania: are women living with HIV more vulnerable? J Acquir Immune Defic Syndr 2014; 67(Suppl. 4):S228-S234.

6. Mesenburg MA, Victora CG, Jacob Serruya S, Ponce de León R, Damaso AH, Domingues MR, Silveira MF. Disrespect and abuse of women during the process of childbirth in the 2015 Pelotas birth cohort. Reprod Health 2018;15(1):54.

7. Tobasía-Hege C, Pinart M, Madeira S, Guedes A, Reveiz L, Valdez-Santiago R, Pileggi V, Arenas-Monreal L, Rojas-Carmona A, Piña-Pozas M, Gómez Ponce de León R, Souza JP. Irrespeto y maltrato durante el parto y el aborto en América Latina: revisión sistemática y metaanálisis. Rev Panam Salud Publica 2019; 43:e36

8. Leite TH, Pereira APE, Leal MC, Silva AAM. Disrespect and abuse of women during birth and postpartum depression: findings from a national survey on childbirth in Brazil. J Affect Disord 2020; 273:391-401.

9. World Health Organization (WHO). The prevention and elimination of disrespect and abuse during facility-based childbirth. Geneva: WHO 2015.

10. Diniz SG, Salgado HO, Andrezzo HFA, Carvalho PGC, Carvalho PCA, Aguiar CA, Niy DY. Violência obstétrica como questão para a saúde pública no brasil: origens, definições, tipologia, impactos sobre a saúde materna, e propostas para sua prevenção. $J$ Hum Growth Dev 2015; 25(3):1-8.

11. Diniz SG, Silveira LP, Mirim LA. Violência contra a mulher: estratégias e respostas do movimento feminista no Brasil (1980-2005). In: Diniz SG, Silveira LP, Mirim LA. Vinte e cinco anos de respostas brasileiras em violência contra a mulher (1980-2005): alcances e limites. 2006. 
12. Leite TH, Carvalho TDG, Marques ES, Pereira APE, Silva AAM, Nakamura-Pereira M, Leal MC. The association between mistreatment of women during childbirth and postnatal maternal and child health care: findings from "Birth in Brazil". Women and Birth 2021; no prelo.

13. Bowser D, Hill K. Exploring evidence for disrespect and abuse in facility-based childbirth report of a landscape analysis [Internet]. Harvard School of Public Health University Research Co., LLC; 2010. [cited 201923 Jan]. Available from: https://www. harpnet.org/resource/exploring-evidence-for-disrespect-and-abuse-in-facility-based-childbirth-report-of-a-landscape-analysis/

14. Bohren MA, Vogel JP, Hunter EC, Lutsiv O, Makh SK, Souza JP, Aguiar C, Saraiva Coneglian F, Diniz AL, Tunçalp Ö, Javadi D, Oladapo OT, Khosla R, Hindin MJ, Gülmezoglu AM. The mistreatment of women during childbirth in health facilities globally: a mixed-methods systematic review. PLoS Med 2015; 12(6):e1001847.

15. Okafor II, Ugwu EO, Obi SN. Disrespect and abuse during facility-based childbirth in a low-income country. Int J Gynaecol Obs 2015;128(2):110-113.

16. Jungari S, Sharma B, Wagh D. Beyond maternal mortality: a systematic review of evidences on mistreatment and disrespect during childbirth in health facilities in India. Trauma Violence Abuse 2021; 22(4):739-751.

17. Freedman LP, Ramsey K, Abuya T, Bellows B, Ndwiga C, Warren CE, Kujawski S, Moyo W, Kruk ME, Mbaruku G. Defining disrespect and abuse of women in childbirth: a research, policy and rights agenda. Bull World Health Organ 2014; 92(12):915-917.

18. Bohren MA, Vogel JP, Fawole B, Maya ET, Maung TM, Baldé MD, Oyeniran AA, Ogunlade M, Adu-Bonsaffoh K, Mon NO, Diallo BA, Bangoura A, Adanu R, Landoulsi S, Gülmezoglu AM, Tunçalp Ö. Methodological development of tools to measure how women are treated during facility-based childbirth in four countries: labor observation and community survey. BMC Med Res Methodol 2018;18(1):132.

19. Coletivo Margarida Alves. Violência obstétrica no abortamento. Coletivo Margarida Alves; 2020.

20. D’Orsi E, Brüggemann OM, Diniz CS, Aguiar JM, Gusman CR, Torres JA, Angulo-Tuesta A, Rattner D, Domingues RM. Social inequalities and women's satisfaction with childbirth care in Brazil: a national hospital-based survey. Cad Saude Publica 2014; 30(Suppl. 1):S154-168

21. Leal MC, Pereira AP, Domingues RM, Theme Filha MM, Dias MA, Nakamura-Pereira M, Bastos MH, Gama SG. Obstetric interventions during labor and childbirth in Brazilian low-risk women. Cad Saude Publica 2014; 30(Suppl. 1):S1-16.
22. World Health Organization (WHO). Intrapartum care for a positive childbirth experience [Internet]. 2018. [cited 201923 Jan]. Available from: http://apps. who.int/iris/bitstream/10665/260178/1/97892415502 15-eng.pdf?ua $=1 \% 0 \mathrm{Ahttp} / /$ www.who.int/reproductivehealth/publications/intrapartum-care-guidelines/ en/

23. Diniz CSG, Rattner D, d'Oliveira AFPL, Aguiar JM, Niy DY. Disrespect and abuse in childbirth in Brazil: social activism, public policies and providers' training. Reprod Health Matters 2018; 26(53):19-35.

24. Leite TH, Pereira APE, Leal MC, Silva AAM. Disrespect and abuse towards women during childbirth and postpartum depression: findings from Birth in Brazil Study. J Affect Disord 2020; 273:391-401.

25. Silveira MF, Mesenburg MA, Bertoldi AD, Mola CL, Bassani DG, Domingues MR, Stein A, Coll CVN. The association between disrespect and abuse of women during childbirth and postpartum depression: findings from the 2015 Pelotas birth cohort study. J Affect Disord 2019; 256:441-447.

26. Souza KJ, Rattner D, Gubert MB. Institutional violence and quality of service in obstetrics are associated with postpartum depression. Rev Saude Publica 2017; 51:69.

27. d'Oliveira AF, Diniz SG, Schraiber LB. Violence against women in health-care institutions: an emerging problem. Lancet 2002; 359(9318):1681-1685.

28. Oliveira S. Por um recorte genealógico da categoria violência obstétrica [Dissertação] Rio de Janeiro: Escola Nacional de Saúde Pública Sergio Arouca, Fundação Oswaldo Cruz; 2019.

29. Leal MC, Szwarcwald CL, Almeida PVB, Aquino EML, Barreto ML, Barros F, Victora Cesar. Saúde reprodutiva, materna, neonatal e infantil nos 30 anos do Sistema Único de Saúde (SUS). Cien Saude Colet 2018 23(6):1915-1928.

30. Pulhez MM. "Parem a violência obstétrica!". Rev Bras Sociol das Emoções 2013; 12(35):544-564.

Artigo apresentado em 23/07/2019

Aprovado em 29/01/2020

Versão final apresentada em 31/01/2020

Editores-chefes: Romeu Gomes, Antônio Augusto Moura da Silva 
